И. В. Гребенник, О. С. Черная, Е. Е. Макарова

Харьковский национальный университет радиоэлектроники, Харьков

\title{
ОПТИМИЗАЦИЯ ЛИНЕЙНЫХ ФУНКЦИЙ НА МНОЖЕСТВЕ ЦИКЛИЧЕСКИХ ПЕРЕСТАНОВОК С ЛИНЕЙНЫМИ ОГРАНИЧЕНИЯМИ
}

\begin{abstract}
Предметом статьи является процесс решения задач дискретной оптимизации на комбинаторных множествах различных классов. Целью является разработка методов решения задачи оптимизации линейной функции с линейными ограничениями на множестве циклических перестановок, погруженном в евклидово пространство. Задачи: найти точное или приближенное решение задачи оптимизации линейной функции с линейными ограничениями на множестве циклических перестановок, погруженном в евклидово пространство. Исследовать свойства задачи оптимизации, оценить приближенное решение. Основные результаты работы. Предложена стратегия решения с использованием алгоритма на основе случайного поиска. Для решения задачи оптимизации линейной функции на множестве циклических перестановок используется подход, основанный на идеологии случайного поиска и аналитическом решении систем линейных неравенств, описывающих ограничения задачи. В процессе решения исходной задачи необходимо многократное решение вспомогательной задачи оптимизации линейной функции на множестве циклических перестановок без ограничений. В работе приводится два подхода к решению вспомогательной задачи. Первый подход позволяет получить точное решение вспомогательной задачи методом ветвей и границ или приближенное решение при использовании дополнительных эвристик с оценкой полученного решения. Второй подход - эвристический метод на основе транспозиций специального вида. Для реализации подхода введен класс транспозиций, представители которого соответствуют критерию смежности в перестановочном многограннике. Предложенные стратегии реализованы программно и протестированы на задачах различной размерности с исходными данными, генерируемыми случайным образом. Проведены вычислительные эксперименты с целью сравнения точности и времени решения исходной задачи методом случайного поиска с использованием предложенных подходов к решению вспомогательной задачи. Выводы. Эксперименты показывают преимущество решения вспомогательной задачи методом ветвей и границ на малых размерностях. При этом на задачах больших размерностей метод на основе транспозиций существенно выигрывает в плане экономии вычислительных мощностей.
\end{abstract}

Ключевые слова комбинаторная оптимизация, линейная функция, линейные ограничения, аналитическое решение системы линейных неравенств, циклические перестановки, случайный поиск, транспозиции.

\section{Введение}

Широкая популярность задач линейной и дискретной оптимизации обусловлена использованием математических моделей этих задач для описания многих фундаментальных и прикладных явлений. В частности ряд популярных задач геометрического проектирования и управления может быть описан различными комбинаторными оптимизационными моделями [1 - 4]. Математические модели таких задач сводятся к соответствующим классам задач комбинаторной оптимизации линейных функций. Области допустимых решений в математических моделях задач геометрического проектирования с дискретными параметрами часто представляются классическими комбинаторными множествами, среди которых множества перестановок, сочетаний, размещений и другие.

Дополнительные линейные ограничения на переменные в таких задачах позволяют выделить следующие известные в литературе классы множеств перестановок: перестановки различных элементов $P_{n}$, перестановки с повторениями, перестановки из $n$ элементов, $k$ из которых различны $P_{n k}$, циклические перестановки $P_{n}^{C}$, перестановки кортежей $P T_{n k}^{m}$, композиции перестановок $P W_{n}$, перестановки содержащие (не содержащие) шаблон (pattern), полиперестановки и другие $[1,4,5,6]$. Существующие подходы к решению задач оптимизации на комбинаторных множествах делятся на две основные группы: методы отсечения и комбинаторные методы. Одним из наиболее распространенных точных комбинаторных методов является метод ветвей и границ, а для решения задач комбинаторной оптимизации большой размерности часто используют методы, основанные на случайном поиске.

Исследование и использование свойств комбинаторных объектов повышает эффективность процессов моделирования и решения задач, имеющих комбинаторную структуру, в частности, задач комбинаторной генерации и комбинаторной оптимизации. Известен способ исследования комбинаторных множеств, основанный на их погружении в евклидово пространство [3, 4]. Тогда выпуклая оболочка образа комбинаторного множества, полученная в результате погружения, представляет собой комбинаторный, в частности, перестановочный, многогранник. Для некоторых подмножеств множества перестановок при погружении в евклидово пространство, на данный момент не описаны соответствующие комбинаторные многогранники. Поэтому при решении задач на этих множествах предлагается использовать перестановочный многогранник, но исследовать те его вершины, которые соответствуют элементам заданного подмножества множества перестановок. Такой подход позволяет воспользоваться одним из базовых свойств перестановочного многогранника - критерием смежности его вершин [7].

В данной работе для решения задач оптимизации на множестве циклических перестановок реали- 
зуется идеология случайного на основе использования свойств циклических перестановок, транспозиций и аналитического решения систем линейных неравенств как ограничений на переменные.

Целью статьи является решение задач оптимизации линейных функций на множестве циклических перестановок с линейными ограничениями.

\section{Основные понятия}

Определение [8]. Линейное упорядочение элементов некоторого порождающего множества $A=$ $A=\left\{a_{1}, a_{2}, \ldots, a_{n}\right\} \quad$ называется перестановкой $\pi=$ $=\pi\left(a_{1}, \ldots, a_{n}\right)=\left(\pi\left(a_{1}\right), \pi\left(a_{2}\right), \ldots, \pi\left(a_{n}\right)\right)=\left(a_{i_{1}}, \ldots, a_{i_{n}}\right)$,

или, если необходимо подчеркнуть тот факт, что она содержит $n$ элементов, $n$-перестановкой. Множество перестановок, порожденное элементами $a_{1}<a_{2}<\ldots<a_{n}$, обозначим $P_{n}$.

Каждый элемент множества $A=\left\{a_{1}, a_{2}, \ldots, a_{n}\right\}$ встречается в единственном цикле перестановки $\pi$, и возможно рассматривать $\pi$ как объединение непересекающихся циклов, или по-другому, как произведение различных циклов $C_{1}, \ldots, C_{k}$, записывая в виде $\pi=C_{1}, \ldots, C_{k}$.

Рассмотрим некоторую перестановку $\pi=\left(\pi\left(a_{1}\right), \pi\left(a_{2}\right), \ldots, \pi\left(a_{n}\right)\right) \in P_{n}, \quad$ и $\quad$ её элемент $\pi\left(a_{i}\right)=a_{j}, \quad \forall i, j \in J_{n}$. Тогда можно записать: $\pi\left(a_{j}\right)=\pi\left(\pi\left(a_{i}\right)\right)=\pi^{2}\left(a_{i}\right)$. Обобщенно можно эту формулу представить в таком виде: $\pi^{k-1}\left(a_{j}\right)=\pi\left(\pi^{k-1}\left(a_{i}\right)\right)=\pi^{k}\left(a_{i}\right), \forall i, j \in J_{n}, k \leq n$.

Таким образом [8], если для некоторого $l \geq 1$ имеем $\pi^{l}\left(a_{i}\right)=a_{i}, \quad i \in J_{n}$, и элементы $a_{i}, \pi\left(a_{i}\right)$, $\pi^{2}\left(a_{i}\right), \ldots, \pi^{l-1}\left(a_{i}\right)$ все различны, то последовательность $\left(a_{i}, \pi\left(a_{i}\right), \pi^{2}\left(a_{i}\right), \ldots, \pi^{l-1}\left(a_{i}\right)\right)$ называется циклом длины $l$.

Определение [9]. Циклической перестановкой называется такая перестановка $\pi$ из $n$ элементов, которая содержит единственный цикл длины $n$, то есть $\pi^{n}\left(a_{i}\right)=a_{i}, \forall i \in J_{n}$. Такие перестановки будем обозначать $\pi_{C}$. Тогда $P_{n}^{C}-$ множество циклических перестановок $\pi_{C}[8,13]$.

Отметим, что множества перестановок $P_{n}$ и циклических перестановок $P_{n}^{C}$ является евклидовыми комбинаторными множествами, или емножествами.

Обозначим $E_{n}^{C}=f\left(P_{n}^{C}\right)$ образ множества циклических перестановок $P_{n}^{C}$ при погружении в евклидово пространство $[3,4,12]$. Осуществим отображение множеств перестановок $P_{n}$ и циклических перестановок $P_{n}^{C}$ в арифметическое евклидово пространство $R^{n}$. Указанное отображение (называемое погружением) зададим в виде:

$$
\begin{gathered}
f: P \rightarrow R^{n}, \forall p=\left(p_{1}, p_{2}, \ldots, p_{n}\right) \in P, \\
x=f(p)=\left(x_{1}, x_{2}, \ldots, x_{n}\right) \in E \subset R^{n}, \\
x_{i}=p_{i}, i \in J_{n} .
\end{gathered}
$$

В результате погружения $f$ каждому множеству $P_{n}, P_{n}^{C}$ поставим во взаимнооднозначное соответствие множество $E \subset R^{n}: \quad E_{n}=f\left(P_{n}\right)$, $E_{n}^{C}=f\left(P_{n}^{C}\right)$.

После погружения множеством $a_{1}<a_{2}<\ldots<a_{n}$ будет порожден перестановочный многогранник $\prod_{n}$, где vert $\prod_{n}=E_{n}$ - его множество вершин.

При решении задач на комбинаторных множествах предлагается использовать перестановочный многогранник и исследовать те его вершины, которые соответствуют элементам заданного подмножества множества перестановок. Одним из базовых свойств перестановочного многогранника является критерий смежности его вершин [10].

Так как между множеством порождающих элементов $A=\left\{a_{1}, a_{2}, \ldots, a_{n}\right\}, a_{1}<a_{2}<\ldots<a_{n}$ и множеством их индексов $J=\{1,2, \ldots, n\}$ существует взаимнооднозначное соответствие, далее, без потери общности, будем рассматривать множество перестановок $P_{n}$, порожденное элементами $\{1,2, \ldots, n\}$, то есть, $A=J_{n}$.

Рассмотрим функцию $\sigma_{i}$ на множестве порождающих элементов, $\sigma_{i}: A \rightarrow A$, определённую следующим образом:

$$
\begin{gathered}
\sigma(1)=1, \sigma(2)=2, \ldots, \sigma(i)=i+1, \\
\sigma(i+1)=i, \ldots, \sigma(n)=n,
\end{gathered}
$$

где $i \in J_{n-1}$. Функция, определенная таким образом, является биективной и представляет собой транспозицию элементов $\{i, i+1\} \subset A[9]$.

Свойством функции $\sigma_{i}$, заданной в виде (2), является то, что она соответствует критерию смежности перестановочного многогранника $\Pi_{n}$. В результате применения функции $\sigma_{i}(p)$ к произвольной перестановке $p \in P_{n}$ будет получена перестановка $p_{k}$, такая что соответствующие $p$ и $p_{k}$ вершины перестановочного многогранника являются смежными.

Для любой вершины $v \in$ vert $\Pi_{n}$ транспозицию $\sigma_{i}(v)$ вида (2) элементов $\{i, i+1\}$, принадлежащих одному циклу длины $k$ соответствующей перестановки $p \in P_{n}$, будем называть транспозицией разрыва. А транспозицию элементов $\{i, i+1\}$, принадлежащих двум разным циклам длины $k_{1}$ и $k_{2}-$ транспозицией соединения. Назовем две транспози- 
ции $\sigma_{i}$ и $\sigma_{j}$ непересекающимися, если подмножества элементов $\{i, i+1\}$ и $\{j, j+1\}$, соответствующие каждой из транспозиций, не пересекаются. Если подмножества $\{i, i+1\}$ и $\{j, j+1\}$, соответствующие двум транспозициям $\sigma_{i}$ и $\sigma_{j}$, пересекаются и $i+1=j$ или $i=j+1$, то такие транспозиции будем называть пересекающимися [11].

\section{Постановка задачи}

Исследуем задачу комбинаторной оптимизации в следующей постановке:

$$
\begin{gathered}
L(p)=\alpha_{i} p_{i} \rightarrow \min ; \\
C p \leq d ; \\
p \in \bar{P} \in\left\{P_{n}, P_{n k}, P_{n}^{C}, P W_{n}, P_{n k}^{m}\right\},
\end{gathered}
$$

где $C=\left[C_{i j}\right]_{m \times n}, d \in R^{n}, \alpha_{i} \in R, p_{i} \in J_{n}=\{1, \ldots, n\}$, $i \in J_{n}, \forall i, j, p_{i} \neq p_{j}$;

$$
\bar{P} \in\left\{P_{n}, P_{n k}, P_{n}^{C}, P W_{n}, P_{n k}^{m}\right\},
$$

где $P_{n}$ - множество перестановок различных элементов, $P_{n k}-$ множество перестановок из $n$ элементов, $k$ из которых различны, $P_{n}^{C}-$ множество циклических перестановок, $P T_{n k}^{m}$ - множество перестановок кортежей, $P W_{n}-$ множество композиции перестановок.

В данной работе далее из множества $\left\{P_{n}, P_{n k}, P_{n}^{C}, P W_{n}, P_{n k}^{m}\right\}$ выберем множество циклических перестановок, то есть $\bar{P}=P_{n}^{C}$. Таким образом, задача состоит в следующем: минимизировать линейную функцию с линейными ограничениями на множестве циклических перестановок.

Используя погружение комбинаторного множества циклических перестановок $P_{n}^{C}$ в евклидово пространство (1), сформулируем эквивалентную задачу оптимизации:

$$
\begin{gathered}
L(x)=\alpha_{i} x_{i} \rightarrow \min ; \\
C x \leq d ; \\
x \in E_{n}^{C} \subset R^{n},
\end{gathered}
$$

где $C=\left[C_{i j}\right]_{m \times n}, d \in R^{n}, \alpha_{i} \in R, x_{i} \geq 0, E_{n}^{C}$ - образ множества циклических перестановок в евклидовом пространстве.

\section{Решение задачи на основе стратегии случайного поиска и метода ветвей и границ}

Для решения задачи оптимизации линейной функции на множестве циклических перестановок используем подход, основанный на идеологии слу- чайного поиска и аналитическом решении систем линейных неравенств, описывающих ограничения задачи. Основа данного подхода была заложена в работе [14] для решения задачи оптимизации линейной функции на множестве перестановок $P_{n}$ с линейными ограничениями.

В дальнейших работах была предложена модификация этого метода для решения задачи (6)(8).Для этого, согласно [14], формируется эквивалентная задача:

$$
\begin{gathered}
L(x)=\alpha_{i} x_{i} \rightarrow \min ; \\
W^{0} x \leq v^{0} ; \\
x_{i} \geq 0, x \in E_{n}^{C} \subset R^{n} x \in E_{n}^{C} \subset R^{n},
\end{gathered}
$$

при решении которой $x_{i}$ находится таким образом:

$$
x_{i}=\arg \min _{x \in E_{n}^{C}}\|x-z(i)\|^{2} .
$$

Для множества перестановок $E_{n}$ нахождение $x_{i}$ вида (12) сводится к нахождению безусловного минимума линейной функции:

$$
x^{*}=\left(x_{1}^{*}, x_{2}^{*}, \ldots, x_{n}^{*}\right)=\arg \min _{x \in E_{n}} \sum_{j=1}^{n} \alpha_{j} x_{j} .
$$

Поскольку множество циклических перестановок является подмножеством множества перестановок, то для любого $x \in E_{n}^{C}$ задача (12) так же сводится к оптимизации линейной функции. Для множества $E_{n}^{C}$ задача (13) не может быть решена на основе упорядочения элементов, и её было предложено решать с помощью метода ветвей и границ [9]. Кроме метода ветвей и границ далее будет предложен альтернативный метод решения задачи (13) с использованием транспозиций особого вида (2).

\section{Решение вспомогательной задачи на основе свойств транспозиций специального вида}

Использование метода ветвей и границ для вспомогательной задачи имеет свои недостатки. Данный метод существенно зависит от исходных данных и при решении задач размерности больше 20 требует задействования вычислительных ресурсов не соизмеримых с получаемой выгодой.

В работе [11] для решения вспомогательной задачи вида (13) была предложена стратегия на основе свойств транспозиций вида (2). Данный алгоритм позволяет получать приближенное решение задачи, но при этом не требует существенных вычислительных ресурсов. Для этого находим перестановку $p^{*} \in P_{n}$, такую что

$$
p^{*}=\arg \min _{p \in P_{n}} \sum_{j=1}^{n} \alpha_{j} p_{j} .
$$


Тогда, если $p^{*} \in P_{n}^{C}$, то решение задачи (13) найдено. В противном случае - необходимо разбиение данной перестановки в произведение циклов. Для этого производится подсчет количества циклов $k$, из которых состоит исходная перестановка. Далее выбираются из всех возможных $n-1$ транспозиций вида (2), $k-1$ транспозиций соединения, таких чтобы с их помощью можно было объединить все $k$ циклов.

Подсчет различных перестановок, которые можно породить с помощью данных транспозиций, путем применения к перестановке $p^{*} \in P_{n}$ выбранных транспозиций, учитывая соотношение пересекающихся и непересекающихся. Далее происходит генерация перестановок и подсчет значения функции цели. В качестве приближенного решения исходной задачи принимается перестановка с минимальным значением целевой функции. В качестве последнего этапа решения исходной задачи осуществляется оценка полученного решения.

В данной работе предлагается модификация метода на основе случайного поиска, при которой решение вспомогательной задачи вида (12) производится с помощью описанного алгоритма, использующего транспозиции вида (2).

\section{Вычислительные эксперименты}

Изложенная стратегия оптимизации линейных функций на циклических перестановках без ограничений реализована программно, проведены вычислительные эксперименты по решению задач вида (6) - (8). Случайным образом генерировались исходные данные задач: коэффициенты функции цели и ограничений, порождающие элементы комбинаторного множества.

Эксперименты проводились в два этапа. На первом этапе решались задачи до 8 переменных, модификацией метода случайного поиска при которой вспомогательная задача решалась с помощью транспозиций порождающих элементов. Далее полученные приближенные решения задачи (6) - (8) сравнивались с решениями, полученными полным перебором.

Случайным образом генерировались коэффициенты двух линейных ограничений, и коэффициенты целевой функции. Коэффициенты целевой функции генерировались в интервале [-200, 200].

Для нахождения решения использовалось количество серий, равное 5 , каждая серия состояла из 10 экспериментальных точек. Рассчитывалась относительная погрешность для задач, в которых результаты решения разработанным методом и методом полного перебора не совпадают. Полученные результаты представлены в табл. 1. Для обозначений выберем следующие сокращения: $N$ - размерность задачи, $S_{M}$ - число совпавших решений, $S_{\bar{M}}$ - число не совпавших решений, $A V G(\Delta)$ - относительная погрешность результатов, $T$ - среднее время решения, секунды.
Таблица 1. Результаты решения задач до 8 переменных

\begin{tabular}{|c|c|c|c|c|}
\hline$N$ & $S_{M}$ & $S_{\bar{M}}$ & $A V G(\Delta)$ & $T$ \\
\hline 3 & 9 & 1 & 0,125 & 0,173 \\
\hline 4 & 10 & 0 & 0 & 0,331 \\
\hline 5 & 9 & 1 & 0,750 & 0,989 \\
\hline 6 & 10 & 0 & 0 & 1,326 \\
\hline 7 & 5 & 5 & 0,714 & 3,512 \\
\hline 8 & 5 & 5 & 0,833 & 4,898 \\
\hline
\end{tabular}

Проведем сравнительную оценку значений, полученных методом случайного поиска и метода ветвей и границ (СП+МВГ) со значениями, полученными методом случайного поиска на основе свойств транспозиций (СП+Т). Результаты представлены в табл. 2.

Таблица 2. Сравнительная оценка значений до 8 переменных

\begin{tabular}{|c|c|c|c|c|}
\hline \multirow{2}{*}{$N$} & \multicolumn{2}{|c|}{$C \Pi+M B \Gamma$} & \multicolumn{2}{c|}{$C \Pi+T$} \\
\cline { 2 - 5 } & $A V G(\Delta)$ & $T$ & $A V G(\Delta)$ & $T$ \\
\hline 3 & 0 & 0,14 & 0,125 & 0,173 \\
\hline 4 & 0,077 & 0,269 & 0 & 0,331 \\
\hline 5 & 0,128 & 0,439 & 0,750 & 0,989 \\
\hline 6 & 0,072 & 0,734 & 0 & 1,326 \\
\hline 7 & 0,084 & 1,527 & 0,714 & 3,512 \\
\hline 8 & 0,134 & 3,402 & 0,833 & 4,898 \\
\hline
\end{tabular}

Обозначим через $A V G(\Delta)$ - относительную погрешность решения.

Исходя из результатов, представленных в табл. 2 можно сделать вывод о том, что задачу оптимизации линейной функции на множестве циклических перестановок на малых размерностях лучше решать методом случайного поиска и методом ветвей и границ. В таком случае при незначительном росте времени вычислений наблюдается так же незначительный рост погрешности. В то время как модификация метода случайного поиска с применением транспозиций показывается относительную погрешность в 8-10 раз большую при соизмеримом времени вычислений.

На втором этапе решались задачи большей размерности. В таких задачах для получения нижней оценки минимума вычислялась две оценки [9] следующего вида:

$$
E_{1}=\left|\frac{E s t-R n d}{R n d}\right|
$$

$$
E_{2}=\left|\frac{E s t-R n d}{E s t}\right|,
$$

где Est - минимум функции цели на циклических перестановках без учета линейных ограничений а Rnd - результат решения задачи методом случайного поиска.

Результаты соответствующих вычислительных экспериментов приведены в табл. 3. Для размерности выше 15 было решено $K$ задач со случайными входными данными. 
Таблица 3. Результаты решения задачи размерности 15-30

\begin{tabular}{|c|c|c|c|c|}
\hline$N$ & $K$ & $A V G\left(E_{1}\right)$ & $A V G\left(E_{2}\right)$ & $T$ \\
\hline 15 & 10 & 0,259 & 0,369 & 15,24 \\
\hline 20 & 10 & 0,454 & 1,571 & 48,86 \\
\hline 30 & 5 & 0,379 & 0,718 & 163,9 \\
\hline
\end{tabular}

Отметим, что с ростом размерности задачи при фиксированном количестве экспериментов характеристики полученного решения ухудшаются. Повышения точности можно добиться путем увеличения количества серий и числа экспериментов в каждой серии.

Сравнительная оценка результатов, полученных методом случайного поиска и методом ветвей и границ со значениями, полученными модификацией метода случайного поиска на основе свойств транспозиций на больших размерностях представлена в табл. 4.

\section{Таблица 4. Сравнительная оценка результатов}

\begin{tabular}{|c|c|c|c|c|c|c|}
\hline \multirow{3}{*}{$N$} & \multicolumn{3}{|c|}{$C \Pi+M B \Gamma$} & \multicolumn{3}{c|}{$C \Pi+T$} \\
\cline { 2 - 7 } & $\begin{array}{c}A V G \\
\left(E_{1}\right)\end{array}$ & $\begin{array}{c}A V G \\
\left(E_{2}\right)\end{array}$ & $T$ & $\begin{array}{c}A V G \\
\left(E_{1}\right)\end{array}$ & $\begin{array}{c}A V G \\
\left(E_{2}\right)\end{array}$ & $T$ \\
\hline 15 & 0,256 & 0,255 & 18,19 & 0,259 & 0,369 & 15,24 \\
\hline 20 & 0,127 & 0,122 & 225,9 & 0,454 & 1,571 & 48,8 \\
\hline 30 & 0,227 & 0,308 & 1805,8 & 0,379 & 0,718 & 163 \\
\hline
\end{tabular}

Исходя из результатов, представленных в табл. 2 и в табл. 4, можно сделать вывод, что на малых размерностях относительная погрешность и среднее время решения модификацией метода случайного поиска на основе свойств транспозиций больше, чем методом случайного поиска и методом ветвей и границ.

На задачах большей размерности, следует отметить, противоположный результат. Это связано с тем, что применение метода ветвей и границ приводит к экспоненциальному росту трудоемкости при увеличении размерности задачи. Кроме того, наблюдается существенная зависимость трудоемкости решения, как от способа ветвления, так и от способа вычисления оценок.

Следует отметить, что метод случайного поиска и метод ветвей и границ при текущих вычислительных мощностях не позволяет решать задачи размерности более 30 , чего нельзя сказать о модификации метода случайного поиска на основе свойств транспозиций.

Результаты решения задач соответствующих размерностей представлены в табл. 5.
Таблица 5. Результаты решения задач больших размерностей

\begin{tabular}{|c|c|c|c|c|}
\hline$N$ & $K$ & $A V G\left(E_{1}\right)$ & $A V G\left(E_{2}\right)$ & $T$ \\
\hline 35 & 2 & 1,602 & 2,661 & 143,5 \\
\hline 40 & 1 & 0,278 & 0,385 & 896,9 \\
\hline 45 & 1 & 0,891 & 8,203 & 325,6 \\
\hline
\end{tabular}

\section{Выводы}

В данной работе реализована стратегия решения задачи оптимизации линейной функции на множестве циклических перестановок, погруженном в евклидово пространство, на основе схемы случайного поиска. Предложены две модификации подхода. Одна из них предполагает нахождение точного решения вспомогательной задачи с помощью метода ветвей и границ. Во второй модификации для вспомогательной задачи находится приближенное решение на основании свойств транспозиций порождающих элементов в перестановках и особенностей комбинаторного множества перестановок. Для реализации подхода введен класс транспозиций, представители которого соответствуют критерию смежности в перестановочном многограннике.

Предложенные стратегии реализованы программно и протестированы на задачах различной размерности с исходными данными, генерируемыми случайным образом. Из результатов вычислительных экспериментов можно сделать вывод что решение задачи оптимизации линейной функции на множестве циклических перестановок с помощью модификации метода случайного поиска на основе свойств транспозиций порождающих элементов выгодно применять для решения задач размерности $N \geq 15$. В таком случае временные затраты на поиск решения существенно ниже чем при решении аналогичных задач методом случайного поиска и методом ветвей и границ. А относительная погрешность существенно не отличается.

Необходимо отметить, что модификация метода случайного поиска на основе свойств транспозиций при решении задач размерности $N>30$ позволяет получить некоторое приближенное решение, в то время как метод ветвей и границ при имеющихся вычислительных мощностях не дает решения задач такой размерности.

Предложенные в данной статье методы могут использоваться для решения задач комбинаторной оптимизации, как на классических комбинаторных множествах, так и на композиционных образах комбинаторных множеств.

\section{СПИСОК ЛИТЕРАТУРЫ}

1. Сергиенко И. В. Математические модели и методы решения задач дискретной оптимизации / И. В. Сергиенко - К.: Наук. думка, 1988. - 472 с.

2. Емец О. А. Комбинаторная оптимизация на размещениях / О. А. Емец, Т. Н. Барболина. - К.: Наук. думка, 2008. $159 \mathrm{c}$.

3. Стоян Ю. Г. Теорія і методи евклідової комбінаторної оптимізації / Ю. Г. Стоян, О. О. Смець - К.: Інститут системних досліджень освіти, 1993. -188 с.

4. Стоян Ю. Г. Математические модели и оптимизационные методы геометрического проектирования / Ю. Г. Стоян, С. В. Яковлев - К.: Наук. думка, 1986. - 268 с. 
5. Гребенник И. В. Упорядочение перестановок при решении задач комбинаторной оптимизации с линейной целевой функцией / И. В. Гребенник, Л. Ю. Юрченко // Системи обробки інформації.- Х.: ХУПС, 2007. - Вип. 8 (66).С. $139-142$.

6. Гребенник И. В. Решение некоторых задач условной оптимизации линейных функций на перестановочном многограннике. / И. В. Гребенник // Радиоэлектроника и информатика.- 1999.- № 1.- С. 55-59.

7. Емеличев В. А. Многогранники, графы, оптимизация. / В. А. Емеличев, М. М. Ковалев, М. К. Кравцов. - М.: Наука, 1981. $-344 \mathrm{c}$.

8. Стенли Р. Перечислительная комбинаторика / Р. Стенли; пер. с англ. А. И. Барвинка. - М.: Мир, 1990. -440 с.

9. Гребенник И. В. Оптимизация линейной функции на множестве циклических перестановок / И. В. Гребенник, А. С.Литвиненко, О. С. Титова. - Бионика Интеллекта. - 2012. - № 2 (79). - С. 8-12.

10. Емец О. А. Решение линейных задач оптимизации на размещениях методом отсечения / О. А. Емец, Т. Н. Барболина // Кибернетика и системный анализ. - 2003. - Т. 37, № 6.

11. Гребенник И. В. Специальные транспозиции элементов перестановок и свойства композиции / И. В. Гребенник, О. С. Черная // Кибернетика и системный анализ.- 2017.- Т. 53, № 1. - С. 79-90..

12. Реклейтис Г. Оптимизация в технике / Г. Реклейтис, А. Рейвиндран, К. Рэгсдел; пер. с англ. В. Я. Алтаева. - М.: Мир, 1986. $-348 \mathrm{c}$.

13. Bona M. Combinatorics of permutations / M. Bona. - Chapman \& Hall/CRC, 2004. - 337 c.

14. Гребенник И. В. Оптимизация линейных функций с линейными ограничениями на комбинаторных множествах на основе случайного поиска / И. В. Гребенник, А. В. Баранов // Искусств. интеллект. - 2007. - № 1.- С. 132-137.

Рецензент: д-р техн. наук, проф. О. О. Можаєв, Національний технічний університет “Харківський політехнічний інститут”, Харків Received (Надійшла) 21.02.2018

Accepted for publication (Прийнята до друку) 28.04.2018

\title{
Оптимізація лінійних функцій на множині циклічних перестановок з лінійними обмеженнями
}

\author{
I. В. Гребеннік, О. С. Чорна, О. Є. Макарова
}

Дана робота присвячена рішенню завдань лінійної і дискретної оптимізації на різних класах комбінаторних множинах. Зокрема в роботі описано рішення задачі оптимізації лінійної функції з лінійними обмеженнями на множині циклічних перестановок. Це стратегія рішення з використанням алгоритму на основі випадкового пошуку. Для рішення задачі оптимізації лінійної функції на множині циклічних перестановок використовано підхід, заснований на ідеології випадкового пошуку і аналітичному рішенні систем лінійних нерівностей, що описують обмеження задачі. У процесі рішення вихідної задачі виникає необхідність багаторазового рішення додаткової задачі лінійної оптимізації на множині циклічних перестановок без обмежень. У роботі наводиться два варіанти рішення додаткової задачі. Перший алгоритм на основі методу гілок і меж. Описано переваги такого підходу - можливість отримати точне рішення, різні варіації методу розгалуження дозволяють гнучко управляти витратами обчислювальних потужностей. Так само в роботі приведена альтернатива методу гілок і меж - евристичний метод на основі транспозицій особливого виду. Для цього було розглянуто клас транспозиція, що характеризується тим, що транспозиції з даного класу відповідають критерію суміжності в переставному багатограннику. Запропоновані стратегії реалізовані програмно і протестовані на завданнях різної розмірності з вихідними даними, що генеруються випадковим чином. Проведено обчислювальні експерименти для порівняння точності і часу рішення вихідної задачі двома варіантами методу випадкового пошуку. Експерименти показують перевагу рішення додаткової задачі методом гілок і меж на малих розмірностях. При цьому на завданнях великих розмірностей метод на основі транспозицій особливого виду істотно виграє в плані економії обчислювальних потужностей.

Ключов і слов а : комбінаторна оптимізація, лінійна функція, перестановки, циклічні перестановки, випадковий пошук, транспозиції.

\section{Optimization of linear functions on a set of cyclic permutations with linear constraints}

\section{Grebennik, O. Chernaya, E. Makarova}

This paper is devoted to the solution of problems of linear and discrete optimization on various classes of combinatorial sets. In particular, the paper describes the solution of the optimization problem for a linear function with linear constraints on the set of cyclic permutations. A decision strategy is described using an algorithm based on random search. To solve the problem of optimizing a linear function on the set of cyclic permutations, we use the approach based on the ideology of random search and the analytical solution of systems of linear inequalities describing the constraints of the problem. In the process of solving the initial problem, it becomes necessary to solve the additional problem of linear optimization multiple times on a set of cyclic permutations without restrictions. The paper presents two options for solving the auxiliary problem. The first algorithm is based on the branch and bound method. Advantages of this approach are - the ability to obtain an exact solution, various variations of the branching method allow flexibly manage the computational costs. An alternative to the branch and bound method is also given in the work - a heuristic method based on transpositions of a special kind. To do this, we considered a class of transpositions characterized by the fact that transpositions from a given class correspond to the adjacency criterion in a permutation polyhedron. The proposed strategies are implemented and tested on problems of different dimensions with initial data generated randomly. Computational experiments have been performed to compare the accuracy and time of the solution of the original problem by two variations of the random search method. Experiments show the advantage of solving an auxiliary problem by the branch and bound method on small dimensions. In large-scale problems, the method based on transpositions of a special kind significantly benefits in terms of saving computational costs.

Keywords: combinatorial optimization, linear function, permutations, cyclic permutations, random search, transpositions. 